\title{
Caracterización clínico-epidemiológica de pacientes con tuberculosis en el Departamento de Caaguazú, Paraguay. 2014 a 2017
}

\author{
Clinical-epidemiological characterization of patients with tuberculosis \\ in the Department of Caaguazú, Paraguay. 2014-2017
}

\begin{abstract}
Gladys Estigarribia1, Oscar Román², Sarita Aguirre³, Guillermo Sequera³, Gloria Aguilar1, Santiago David Toledo Nuñez5, Aldo Rodríguez, Carlos Miguel Ríos-González 4 , Pablo Martínez ${ }^{4}$, Julieta María Méndez Romero ${ }^{1}$ y Melissa Patricia Valdez Benítez ${ }^{3}$
\end{abstract}

'Universidad Nacional de Caaguazú, Instituto Regional de Investigación en Salud, Paraguay.

2Programa Nacional de Lucha contra la Tuberculosis, Ministerio de Salud Pública y Bienestar Social, Asunción.

${ }^{3}$ Dirección de Vigilancia de la Salud, Ministerio de Salud Pública y Bienestar Social, Asunción.

${ }^{4}$ Facultad de Ciencias Médicas, Universidad Nacional de Caaguazú, Paraguay.

${ }^{5}$ Universidad Nacional de Asunción.

Los autores declaran la ausencia de conflictos de interés.

Estudio autofinanciado.

Recibido: 4 de marzo de 2020 / Aceptado: 11 de noviembre de 2020

\section{Resumen}

Introducción: La tuberculosis (TBC) es una de las diez principales causas de muerte en todo el mundo. Objetivo: Caracterizar clínica y epidemiológicamente los casos de TBC del Departamento de Caaguazú-Paraguay, entre los años 2014 y 2017. Pacientes y Métodos: Se realizó un estudio observacional, retrospectivo, utilizando datos secundarios del Programa Nacional de Control de la Tuberculosis (PNCT). La población: 659 casos de TBC registrados en el PNCT. Las variables: edad, sexo, grupo poblacional, tipo de TBC, co-infección TBC/VIH, y categoría de egreso. Procesamos la base de datos en Excel $2016^{\circ}$ usando Stata $14.0^{\circledR}$. Resultado: El 63,3\% de los participantes fue del género masculino. La edad promedio fue de 35,8 años. El 39,6\% eran indígenas y $14,5 \%$ fueron personas privadas de libertad (PPL) conocidos como reclusos. El $89,6 \%$ tuvo TBC pulmonar, $2,4 \%$ tuvo coinfección TBC/VIH. La tasa de incidencia fue superior a 21,6/100.000 habts en 2014. La incidencia en indígenas fue de 76,5/100.000 habts en 2017. La incidencia en PPL fue de 2.272,1/100.000 habitantes en 2017. Conclusión: La incidencia de TBC en el Departamento de Caaguazú es baja en la población general afectando principalmente a hombres, mientras que en la población indígena y PPL es alta.

Palabras clave: tuberculosis; Mycobacterium; epidemiología; determinantes sociales de la salud.

\section{Abstract}

Background: Tuberculosis (TB) is one of the ten leading causes of death worldwide. Aim: To characterize the clinical and epidemiological point of view of TB cases reported in the Department of CaaguazúParaguay, from 2014 to 2017. Methods: Observational, descriptive, retrospective study; Population: 659 cases of TB registered in the National Tuberculosis Control Program (NTCP); variables: age, sex, population group, type of TB, TB/HIV coinfection. We procesed database in Excel $2016^{\circ}$ using Stata $14.0^{\circledR}$. Results: $63.3 \%$ were of male gender, average age: 35.8 years, $39.6 \%$ were indigenous and $85.4 \%$ were liberty deprived persons known as inmates (LDP), $89.6 \%$ had pulmonary TB and $2,4 \%$ had TB/HIV coinfection. Incidence rate exceed 21.6/100,000 inhabitants in 2014. Indigenous incidence was 76.5/100,000 inhabitants in 2017, LDP incidence was 2,272.1/ 100,000 inhabitants in 2017. Conclusion: The incidence of TB in the Department of Caaguazú is low, mainly affecting men, while TB incidence in indigenous people and LDP was high.

Keywords: tuberculosis; Mycobacterium sp; epidemiology; social determinants of health. 


\section{Introducción}

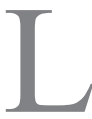

a tuberculosis (TBC) es una de las diez principales causas de muerte en todo el mundo; a su vez, implica una gran inversión en salud pública con escaso impacto en términos de control efectivo $(9,8 \mathrm{del}$ PIB) afectando principalmente a las regiones en vías de desarrollo $^{1}$. En el año 2018, mundialmente se estimaron 10 millones de casos nuevos de TBC. Globalmente, 1.2 millones (rango, 1,1-1,3 millones) de muertes por TBC entre personas seronegativas para VIH y un adicional de 251.000 muertes (rango 223.000-281.000) entre personas infectadas por VIH. De todos los casos de TBC 57\% corresponde a hombres, $32 \%$ mujeres y $11 \%$ niños $^{2,3}$.

En el Paraguay, un país de 6.956.000 habts., se ha notificado al sistema de información del Programa Nacional de Control de la Tuberculosis (PNCT) en el año 2016 un total de 2.436 casos de TBC y una incidencia de 35,6 casos por 100.000 habts. La mayor cantidad de casos se registró en Central, Alto Paraná y Asunción. Las incidencias más altas se encuentran en Pte. Hayes, Boquerón y Alto Paraguay ${ }^{4}$. Según los últimos datos publicados de la OMS, la incidencia de TBC en el Paraguay tiene una tendencia decreciente de 66 a 44/100.000 habts. en el periodo de 1990 al 2013. La incidencia en población indígena en el año 2013 fue de 272/100.000 habts. La tasa de mortalidad reportada en el 2016 fue de 4 por 100.000 habts. $(3,5 \text { a } 4,6)^{5,6}$.

Los objetivos y metas globales para la reducción de la TBC en el mundo forman parte de los Objetivos de Desarrollo sostenible (ODS) y la estrategia End TB de la Organización Mundial de la Salud. En este contexto, en el periodo 2000 al 2018 se observó una reducción de 1,6\% por año de la tasa de incidencia y una reducción acumulativa desde 2015 a 2018 de solamente 6,3\%. La reducción global de número de muertes entre 2015 y 2018 fue de $11 \% \%^{7-9}$.

El PNCT está integrado en la red de Servicios de Salud. En el año 2000, el Paraguay implementó la estrategia de la OMS: Tratamiento Acortado Estrictamente Supervisado (DOTS/TAES)*, comenzando su expansión en seis de las 18 regiones sanitarias del Paraguay en el año 2002 En diciembre de 2004, se obtuvo el primer subsidio del Fondo Mundial, con el fin de incrementar la cobertura de la estrategia DOTS/TAES en las 18 regiones sanitarias del país. El número de laboratorios que realizan baciloscopias se ha incrementado de 34 en el 2003 a 151 en el 2013. El equipo técnico de TBC y los equipos regionales han adquirido capacidades y competencias para realizar supervisiones a nivel regional y local, lo cual contribuyó a mejorar la implementación de la estrategia DOTS/TAES, principalmente en los componentes de detección, TAES y sistema de registro ${ }^{5}$.

\footnotetext{
*DOTS: del inglés Direct Observed Treatment Short-course.
}

Considerando que en el Paraguay se cuenta con escasos estudios que caracterizan la epidemia y analizan datos sub-nacionales, es necesario determinar las características epidemiológicas y clínicas de los pacientes con TBC en la región de Caaguazú, de manera que les permita a las autoridades y directivos regionales de salud enfrentar los actuales y los nuevos desafíos para alcanzar las metas propuestas al 2020, referente a la disminución de la incidencia y mortalidad por TBC. Por lo que se planteó el objetivo de caracterizar clínica y epidemiológicamente los casos de TBC pertenecientes al Departamento de Caaguazú-Paraguay, entre los años 2014 y 2017.

\section{Metodología}

\section{Diseño del estudio}

Estudio observacional, retrospectivo. Se utilizaron registros oficiales del PNCT, Ministerio de Salud Pública y Bienestar Social, del período entre enero de 2014 y diciembre de 2017.

\section{Criterio de inclusión}

Se incluyen todos los casos que ingresan a tratamiento según la nomenclatura sugerida por la $\mathrm{OMS}^{8}$ :

- Caso de TBC bacteriológicamente confirmado: persona con una muestra biológica positiva por baciloscopia, cultivo o prueba rápida como el GeneXpert

- Caso de TBC clínicamente diagnosticado: es aquel que no cumple los criterios de confirmación bacteriológica (baciloscopia negativa, sin resultado de baciloscopia o GeneXpert que no detecta Mycobacterium tuberculosis, pero es diagnosticado por un médico o proveedor quien ha decidido dar un ciclo completo de tratamiento. Esta definición incluye casos diagnosticados sobre la base de anomalías en radiografías o histología sugestiva (anatomía patológica) y casos extra-pulmonares sin confirmación de laboratorio.

Estos son los criterios de la Guía del PNCT de Paraguay que se encuentran en la Guía de Tuberculosis 5 .

\section{Criterio de exclusión}

Casos con pérdida de más de $20 \%$ de los datos de las variables de interés.

\section{Variables}

- TBC pulmonar (TBCP): TBC bacteriológicamente confirmada o clínicamente diagnosticada, que afecta al parénquima pulmonar y el árbol traqueo- bronquial. La TBC miliar se clasifica como TBCP debido a que las lesiones comprometen el parénquima pulmonar. Un paciente con TBCP y extra-pulmonar debe clasificarse como un caso de TBCP. 
- TBC extra-pulmonar (TBCEP): TBC bacteriológicamente confirmada o clínicamente diagnosticada, que involucra órganos distintos a los pulmones; por ejemplo, pleura, ganglios linfáticos, abdomen, tracto genito-urinario, piel, articulaciones, huesos, meninges y otros.

- Paciente con TBC y VIH: Caso bacteriológicamente confirmado o clínicamente diagnosticado, con un resultado positivo de la prueba para VIH.

- Paciente con TBCy sin VIH: Caso bacteriológicamente confirmado o clínicamente diagnosticado, con resultado negativo de la prueba para VIH.

- Paciente curado: Paciente con TBCP con bacteriología confirmada al inicio del tratamiento:

- Si fue confirmado inicialmente por baciloscopia, debe contar con una baciloscopia negativa al completar el último mes de tratamiento y al menos en una ocasión anterior durante el desarrollo del ciclo del tratamiento.

- Si fue confirmado inicialmente por cultivo y/o GeneXpert, debe contar con por lo menos un cultivo negativo durante el tratamiento y una baciloscopia negativa al final del último mes de tratamiento.

- Tratamiento completo: Paciente con TBC que completó el tratamiento, sin evidencia de fracaso. Esta categoría de egreso es utilizada también en las formas extra-pulmonares y casos con baciloscopia (-) al inicio de tratamiento o clínicamente diagnosticado

- Fallecido: Paciente con TBC que muere, por cualquier razón, antes de iniciar o durante el curso del tratamiento.

- Pérdida de seguimiento: Paciente con TBC que no inició tratamiento o interrumpió el tratamiento durante al menos un mes o más.

- Fracaso del tratamiento: Paciente con TBC cuya baciloscopia o cultivo de esputo es positivo en el mes 5 o de allí en adelante, durante el tratamiento.

\section{Variables analizadas}

- Datos filiatorios: sexo (masculino/femenino), grupo de edad ( $\leq 19$ años, 20 a 39 años, 40 a 59 años, 60 y más años), área de residencia (urbana/rural), considerando como área rural al conjunto de centros poblados con menos de 2.500 habitantes.

- Datos sociográficos: perteneciente a la población indígena (si/no), privada de su libertad (sí/no). Los datos se obtuvieron de los registros oficiales.

- Clasificación según localización anatómica: TBCP y extra-pulmonar.

- Clasificación según co-infección por VIH: Paciente con TBC e infección por VIH /Paciente con TBC y sin infección por VIH.

- Método de diagnóstico: baciloscopia, cultivo, GenXpert.
- Categoría de egreso: fallecido, fracaso al tratamiento, pérdida de seguimiento, tratamiento completo, curado.

Los datos fueron extraídos de la base de datos del PNCT y tienen como fuente el "Formulario 2". Ficha de Notificación y Seguimiento del Caso de TBC el que es llenado y posteriormente cargado al citado sistema. El formulario es el oficial del Plan Estratégico de la Respuesta Nacional a la Tuberculosis en Paraguay (MSPyBS) al cual notifican todas las unidades notificadoras del país, (notificación obligatoria) . $^{5}$

\section{Análisis estadístico}

Se empleó el paquete estadístico Stata 14.0., realizando controles de calidad de datos: eliminación de duplicados e inconsistencia. Para la descripción de las variables cualitativas se utilizaron distribución de frecuencias absolutas y porcentuales. Para el análisis espacial y la representación cartográfica final, fue exportada la base de datos desde el software Mapinfo al formato compatible con el SigEpi 1.42000 . Se combinaron las técnicas de procesamiento estadístico que ofrecen el SigEpi 1.4 y el Stata 14.0. Para la variable de interés fue elaborado un mapa temático, en el SigEpi 1.4, con el objetivo de visualizar y analizar la distribución espacial y la frecuencia de ésta.

Se consideró una incidencia de TBC baja si el valor fue menor a 25 , moderada entre 25 y 49 , alta entre 50 y 84 y muy alta cuando fue mayor a 85 .

El protocolo de este trabajo fue evaluado y aprobado por el Comité de Ética en Investigación de la Facultad de Ciencias Médicas de la Universidad Nacional de Caaguazú. Se solicitó el permiso correspondiente para la utilización de las bases de datos al MSPyBS -una base anónima- por lo cual se respetó la confidencialidad firmando un acuerdo.

\section{Aspectos éticos-regulatorios}

Toda la información recolectada fue confidencial, por parte de los investigadores no se repitió, falsificó o crearon datos.

\section{Resultados}

Ingresaron al estudio 659 personas del Departamento de Caaguazú desde enero de 2014 hasta diciembre de 2017. Las tasas de incidencia de $\mathrm{TBC}$ en la región de Caaguazú fueron 22,49 y 41,03/100.000 habts. en los años 2014 y 2017, respectivamente, con un incremento de $82 \%$ en relación al 2014 y un incremento promedio anual de $22 \%$. En cuanto a población indígena, los datos fueron muy superiores, alcanzando valores de 76,5/100.000 habts. en el último año del estudio en el Departamento de Caaguazú, habiendo sido de 46,2/100.000 habts. en el 
Tabla 1. Características socio-demográficas de los pacientes diagnosticados con tuberculosis, Caaguazú, Paraguay, 2014 a 2017. $n=659$

\begin{tabular}{|c|c|c|}
\hline & $\mathbf{n}$ & $\%$ \\
\hline \multicolumn{3}{|l|}{ Sexo $(n=659)$} \\
\hline Femenino & 241 & 36,5 \\
\hline Masculino & 418 & 63,4 \\
\hline $\operatorname{Edad}(n=622)$ años & 39 (Cl 26-55) & \\
\hline \multicolumn{3}{|c|}{ Área de residencia $(n=492)$} \\
\hline Urbana & 89 & 18,0 \\
\hline Rural & 403 & 81,9 \\
\hline \multicolumn{3}{|l|}{ Población ( $n$ = 659) } \\
\hline Indígena & 261 & 39,6 \\
\hline No indígena & 398 & 60,3 \\
\hline \multicolumn{3}{|l|}{ Etnias indígenas (261) } \\
\hline Ache & 33 & 12,6 \\
\hline Mbya & 221 & 84,6 \\
\hline Ava Guaraní & 7 & 2,6 \\
\hline \multicolumn{3}{|c|}{ Privado de libertad $(n=412)$} \\
\hline Sí & 60 & 14,5 \\
\hline No & 352 & 85,4 \\
\hline
\end{tabular}

Tabla 2. Características clínicas y de laboratorio de pacientes diagnosticados con tuberculosis, Departamento de Caaguazú, Paraguay, 2014 a 2017. $\mathrm{n}=659$

\begin{tabular}{lrr}
\hline \multicolumn{1}{c}{$\mathbf{n}$} & $\%$ \\
\hline Tipo de tuberculosis $(\mathrm{n}=659)$ & & \\
Pulmonar & 591 & 89,6 \\
Extra-pulmonar & 44 & 6,6 \\
Ambos & 24 & 3,6 \\
\hline Co-infección de TBC/ VIH $(\mathrm{n}=413)$ & & \\
$\quad$ No reactivo & 403 & 97,5 \\
Reactivo & 10 & 2,4 \\
\hline Método de confirmación $(\mathrm{n}=516)$ & & \\
Baciloscopia & 272 & 52,7 \\
Cultivo & 19 & 3,6 \\
GenXpert & 41 & 7,9 \\
Clínica, Rx, TC, otros & 184 & 35,6 \\
\hline Categoría de egreso $(\mathrm{n}=466)$ & & \\
Fallecido & & \\
Fracaso al tratamiento & 44 & 9,4 \\
Traslado & 2 & 0,4 \\
Perdida en el seguimiento & 4 & 0,8 \\
No evaluado & 79 & 16,9 \\
Tratamiento completo & 1 & 0,2 \\
Curado & 182 & 39,0 \\
& 154 & 33,0 \\
\hline
\end{tabular}

año 2014. En Paraguay, la tasa de incidencia general en el 2014 fue de 46,2 decreciendo a 39,8/100.000 habts. en el 2017. Las tasas de incidencia en población privada de libertad (PPL) han alcanzado valores de 2.272,1/100.000 habts. en el año 2017 (Figura 1).

El 63,3\% de los pacientes perteneció al género masculino. Alrededor de la quinta parte de la de los casos reside en zonas urbanas (n: 89; 18,09\%). Entre los sujetos, $261(39,6 \%)$ pertenecían a alguna parcialidad indígena y la mayoría $(85,4 \%)$ al momento del diagnóstico no se encontraba privado de su libertad (Tabla 1).

La mayoría de los sujetos, correspondientes a 591 $(89,6 \%)$ presentó una TBCP.

Sólo en 10 sujetos se registró co-infección TBC/VIH $(2,4 \%)$.

El método de confirmación de la TBC más utilizado fue la baciloscopia (52,7\%).

El 39\% egresó con un tratamiento completo y $33 \%$ se consideró curado (Tabla 2).

\section{Distribución regional de la incidencia}

El Departamento de Caaguazú cuenta con 22 distritos, de los cuales Coronel Oviedo y Caaguazú tienen una población de 121.126 y 126.477 habts., respectivamente Se comparó la incidencia regional de TBC del año 2014 con el año 2017. Las áreas amarillas son zonas de baja incidencia, las de color naranja oscuro de mediana incidencia, las áreas color naranja claro de alta incidencia y las áreas rojas muy alta incidencia. Como se observa predominan zonas de alta incidencia, que incluso progresan a muy alta incidencia (Figura 2).

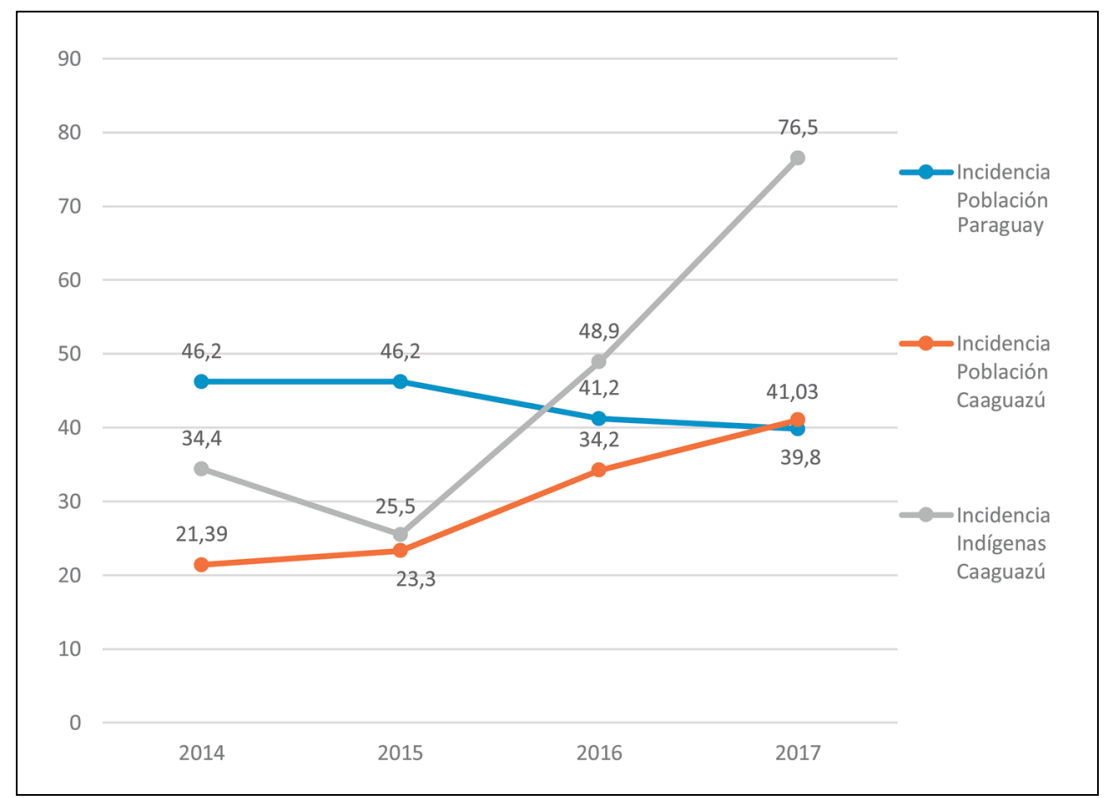

Figura 1. Incidencia de tuberculosis en Paraguay y en población total e indígenas del Departamento de Caaguazú. Años 2014-2017. 
Figura 2. Distribución geoespacial de la incidencia de tuberculosis en el Departamento de Caaguazú, Paraguay, 2014 a 2017. $\mathrm{N}=659$.
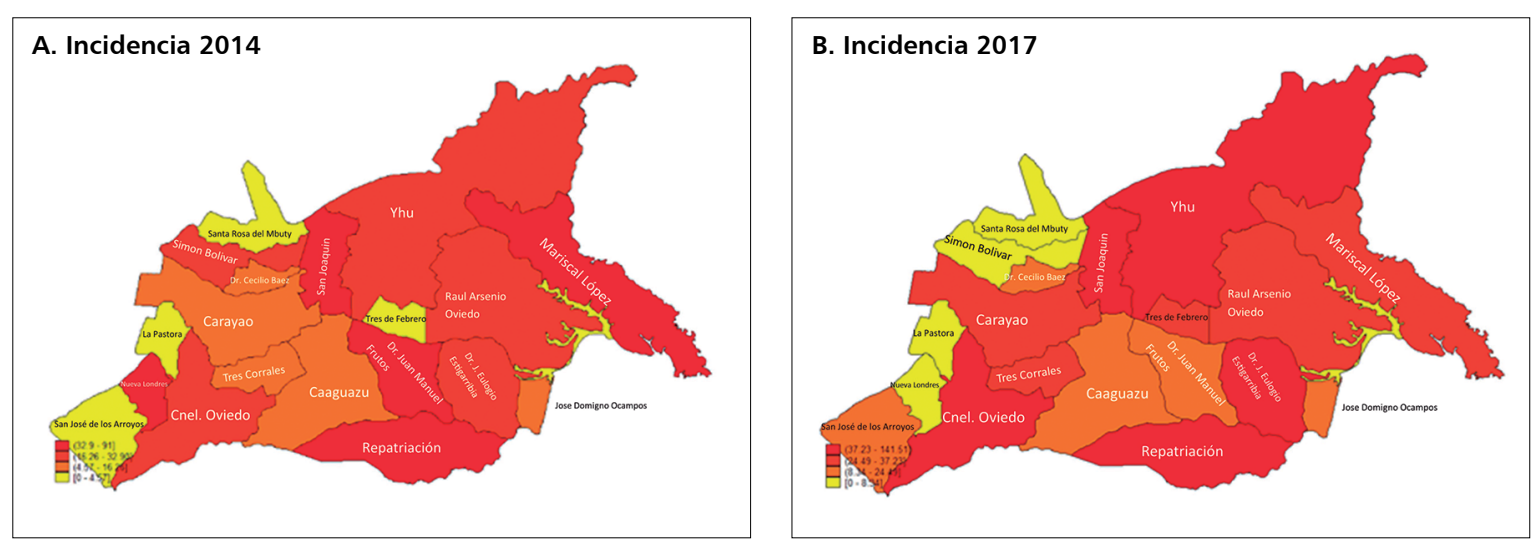

\section{Discusión}

Este es el primer estudio en caracterizar epidemiológica y clínicamente a las personas con TBC en la región de Caaguazú. La carga de la TBC en la región representa $8 \%$ de la carga global de la TBC en el Paraguay.

La tasa de incidencia de TBC en Caaguazú reportada para el 2017 de 41,30/100.000 habts. fue mayor que la incidencia de 39,8 casos por 100.000 habts. reportada a nivel nacional, situación que amerita el análisis de la situación regional y la evaluación de los factores de riesgo para las nuevas infecciones de TBC en esta región.

Los datos en población indígena en el Departamento de Caaguazú fueron muy superiores alcanzando valores de $76,5 / 100.000$ habts. en el último año del estudio. Por otra parte, las tasas de incidencia en PPL alcanzaron valores de 2.272,1/100.000 habts. en el año 2017. Por lo que las acciones sanitarias dirigidas a estos grupos de riesgo deben ser más oportunas y articularse con otros sectores, a fin de reducir la vulnerabilidad de los mismos a la TBC.

A pesar que la incidencia de la enfermedad en todas sus formas ha disminuido progresivamente en la población indígena $^{10,11}$, todavía los valores en dicha población duplican a los valores en la población en general. En América, la incidencia de TBC en indígenas es nueve veces mayor en relación a la población general, así mismo en PPL ${ }^{11,12}$. Los indígenas de nuestro país enfrentan desigualdades, vinculadas a los determinantes estructurales de acceso a la salud y educación, que los sitúan en una posición de mayor vulnerabilidad ante la población no indígena. Si bien se han fortalecido las unidades de salud familiar, igual el acceso a la salud de esta población sigue siendo un gran desafío para el país.

La casi nula cobertura sanitaria a las comunidades indígenas ha deteriorado aún más sus condiciones de vida. La ausencia de una asistencia activa a los casos infecciosos, la poca accesibilidad por causas geográficas, económicas, lingüísticas o culturales de los indígenas a los centros asistenciales, la falta de seguimiento ambulatorio a los pacientes indígenas y el frecuente abandono de los tratamientos, favorecen la permanencia de enfermedades como la TBC en la población indígena.

En este estudio y en el mundo, los hombres representan 2/3 de los casos, siendo igual que en México. En Colombia, la mayoría de los pacientes con TBC también fueron hombres jóvenes ${ }^{13}$. En los municipios amazónicos de la frontera de Brasil-Colombia-Perú-Venezuela, la TBC afectó principalmente a pueblos indígenas $(51,9 \%)$, hombres $(57,9 \%)$ y personas de 25 a 44 años $(31,4 \%)^{14}$.

La mayoría de los sujetos 591 (89,68\%) presentó TBC pulmonar. La co-infección de TBC/VIH se presentó en $2,4 \%$ de los casos.

La baciloscopia sigue siendo el método más empleado para el diagnóstico, seguido del método clínico con medios auxiliares ( $\mathrm{Rx}, \mathrm{TC}$ y otros). Según la OMS es necesario el hecho de contar con políticas nacionales que impulsen la implementación de pruebas rápidas a todos los casos presuntivos.

En cuanto a la categoría de egreso, $33 \%$ se curó, $39 \%$ realizó tratamiento completo, $9,4 \%$ falleció, y $0,4 \%$ fracasó al tratamiento. En cuanto a los municipios amazónicos de la frontera de Brasil-Colombia-Perú-Venezuela; en $70,0 \%$ de los casos notificados, los pacientes fueron dados de alta como curados. El incumplimiento del tratamiento se registró en el $10,0 \%$ de los pacientes.

De todas las muertes, $4,1 \%$ fue por TBC y otras causas, y $1,7 \%$ por TBC multi-resistente ${ }^{14}$. En las Américas los parámetros son $75,4 \%$ de éxito, $7,3 \%$ de fallecidos y $0,5 \%$ de fracaso ${ }^{15,16}$. Se deben reforzar las acciones, a fin de mejorar los parámetros de éxito y disminuir la tasa de fallecidos. Primeramente, reforzar el Programa de TBC, instalar este programa en todas las unidades de salud, dedicando tiempo al personal y verificando su capacitación. Además, buscar socios de colaboración en el intersector y con el resto del equipo de salud. Debe realizarse esfuerzos para llegar a un diagnóstico precoz y a un completo estudio de contactos ampliado. Se debe estimular el diagnóstico de infección por VIH que puede 
todo, para el trabajo de campo la logística es muy limitada. En el caso de TBC, hay apoyo externo del Fondo Mundial para el fortalecimiento de la red de laboratorios en regiones con comunidades indígenas. En la región de Caaguazú específicamente, se encuentra centralizado en un servicio y las muestras llegan a veces tarde y sin posibilidades de procesamiento por el tiempo transcurrido. Existen intervenciones puntuales no regulares con un presupuesto limitado.

Las acciones del programa de TBC en Paraguay son gratuitas y se resumen en la búsqueda de casos presuntivos, seguimiento de casos, control de contactos y provisión de medicamentos.

La resistencia a fármacos antituberculosos en la población indígena es un problema en nuestro Departamento. Deben propiciarse proyectos de midan adherencia a la terapia y evidencien problemas que puedan abordarse de manera direccionada en base a las evidencias encontradas, en módulos educativos a las comunidades, junto con supervisión y visitas domiciliarias; si bien las supervisiones y visitas domiciliares se realizan, la cobertura no llega siquiera al $50 \%$.

Este trabajo demuestra la necesidad de realizar intervenciones sobre grupos de riesgo caracterizados, permitiendo focalizar recursos y esfuerzos en el control de la de la TBC en Paraguay.

\section{Referencias bibliográficas}

1.- OMS. Países. Paraguay. 2016. https://www. who.int/countries/pry/es/

2.- Las 10 principales causas de defunción https:// www.who.int/es/news-room/fact-sheets/detail/ the-top-10-causes-of-death

3.- OMS | Tuberculosis WHO. Octubre 2019 https://www.who.int/es/news-room/fact-sheets/ detail/tuberculosis

4.- OPS Paraguay - Plan Nacional de Control de la Coinfección Tuberculosis-VIH 2017-2020| OPS. Pan American Health Organization. octubre 2017 https://www.paho.org/par/ index.php?option $=$ com_docman\&task $=\mathrm{doc}$ download\&gid $=577$.

5.- $\quad$ MSPyBS Paraguay. Plan estratégico de la Respuesta Nacional a la tuberculosis en Paraguay. 2016. http://vigisalud.gov.py/ webdgvs/views/paginas/webtb/docs/plan estrategico_nacional_tb.pdf

6.- WHO. Tuberculosis Profile: Paraguay. 2018. https://worldhealthorg.shinyapps.io/ tb_profiles/?_inputs_\&lan=\%22EN\% $\% 22 \&$ iso $2=$ $\% 22 \mathrm{PY} \% 22 \&$ main_tabs $=\% 22$ est_tab\%22.

7.- Rodríguez M. OPS/OMS Paraguay - Países de las Américas pueden poner fin a la tuberculosis en 2030 si se aceleran las acciones necesarias para alcanzar esta meta $\mid$ OPS/OMS. Pan American Health Organization / World Health Organization. 2019 https://www.paho.org/par/ index.php?option $=$ com_content $\&$ view $=$ article $\&$ $\mathrm{id}=2130$ :paises-de-las-americas-pueden-ponerfin-a-la-tuberculosis-en-2030-si-se-aceleranlas-acciones-necesarias-para-alcanzar-estameta\&Itemid $=258$

8.- WHO. Definiciones y marco de trabajo para la notificación de tuberculosis. 2013. Disponible en: https://www.who.int/tb/publications/ definitions/es/

9.- MacNeil A, Glaziou P, Sismanidis C, Maloney S, Floyd K. Global epidemiology of tuberculosis and progress toward achieving global targets - 2017. MMWR Morbid Mortal Wkly Rep. 2019 March 22; 68(11): 263-6. doi: 10.15585 / mmwr.mm6811a3

10.- OMS | Estrategia Fin de la OMS para poner fin a la tuberculosis WHO. Mayo 2014. https:// www.who.int/tb/strategy/end-tb/es/

11.- Benitez, J. Prevalencia de tuberculosis en indígenas de Caaguazú, 2015 a 2017. 2018. Tesis Doctoral. FCM-UNCA. https://repositorio. fcmunca.edu.py/xmlui/handle/123456789/38.

12.- Ministerio de Salud Pública y Bienestar Social. Incidencia por tuberculosis en Paraguay, con tendencia decreciente - Ministerio de Salud Publica y Bienestar Social https://www.mspbs. gov.py/portal/8212/incidencia-por-tuberculosisen-paraguay-con-tendencia-decreciente.html

13.- Rojas C M, Villegas S L, Piñeros H M, Chamorro E M, Durán C E, Hernández E L, et al. Características clínicas, epidemiológicas y microbiológicas de una cohorte de pacientes con tuberculosis pulmonar en Cali, Colombia. Biomedica. 2011; 30 (4): 482. doi: https://doi. org/10.7705/biomedica.v30i4.286.

14.- Belo E N, Orellana J D Y, Levino A, Basta P C. Tuberculosis in Amazonian municipalities of the Brazil-Colombia-Peru-Venezuela border: epidemiological situation and risk factors associated with treatment default. Rev Panam Salud Publica, 2013; 34 (5): 321-30. PMID: 24553759.

15.- Organización Panamericana de la Salud. Situación de la tuberculosis en las Américas, 2016 [Internet]. Washington D.C.: Organización Panamericana de la Salud; 2016. https://www. paho.org/hq/dmdocuments/2017/2017-chahoja-info-situacion-tb-americas.pdf.

16.- Organización Mundial de la Salud, Organización Panamericana de la Salud. Tuberculosis en las Américas [Internet]. Washington, D.C; 2018 Report No.: OPS/ CDE/18-036. http://iris.paho.org/xmlui/ bitstream/handle/123456789/49510/ OPSCDE18036_spa? sequence $=2 \&$ isAllowed $=\mathrm{y}$

17.- Heredia-Navarrete M R, Puc-Franco M, Caamal-Ley Á, Vargas-González A. Determinantes sociales relacionados con el tratamiento de tuberculosis en Yucatán, México. 2012; 23(3):113-20. http://revistabiomedica. $\mathrm{mx} /$ index.php/revbiomed/article/view/81. DOI: https://doi.org/10.32776/revbiomed.v23i3.81. 